\title{
Coercive and anisotropy fields in patterned amorphous FeSi submicrometric structures
}

\author{
M. Vélez, R. Morales, and J. M. Alameda \\ Dpto. Fisica, Fac. Ciencias, Universidad de Oviedo, c/Calvo Sotelo s/n, 33007 Oviedo, Spain \\ F. Briones \\ CNM-CSIC, IMM, Isaac Newton 8, Tres Cantos, Madrid, Spain \\ J. I. Martín and J. L. Vicent \\ Dpto. Fisica de Materiales, Universidad Complutense, 28040 Madrid, Spain
}

\begin{abstract}
Amorphous $\mathrm{Fe}_{x} \mathrm{Si}_{1-x}$ films have been prepared on $\mathrm{Si}$ substrates in order to fabricate submicrometric magnetic structures with soft magnetic behavior. The magnetic properties compositional dependence of the unpatterned samples has been analyzed to select the Fe content $(x=0.7)$ with the lowest coercive and anisotropy fields values. Arrays of $\mathrm{Fe}_{0.7} \mathrm{Si}_{0.3}$ lines have been fabricated by electron beam lithography combined with a liftoff technique, with typical dimensions of $200 \mathrm{~nm}$ linewidth and $1 \mu \mathrm{m}$ line spacing. These arrays present coercive fields parallel to the line direction as small as 9 Oe. (C) 2000 American Institute of Physics. [S0021-8979(00)38808-9]
\end{abstract}

\section{INTRODUCTION}

Ordered magnetic structures of submicrometric size are attracting a lot of interest in the field of Material Science. They allow us to study the physical properties of the material in a controlled geometry and reduced dimensions, and they also provide a good tool to control the material behavior in the small scale needed for future applications in magnetic recording technology. Recently, different lithographic techniques have been developed in order to pattern magnetic materials in this small scale, such as x-ray lithography, ${ }^{1}$ laser interference lithography, ${ }^{2}$ scanning tunneling microscopy assisted techniques, ${ }^{3}$ and electron beam lithography. ${ }^{4}$

In this way, different kinds of magnetic arrays of lines and particles have been fabricated in the last few years to analyze the influence of the reduced dimensions in fundamental magnetic properties as, for example, magnetization reversal processes ${ }^{5,6}$ or domain wall magnetoresistance. ${ }^{7}$ Most of these studies have been performed in samples made of pure transition metals or NiFe alloys either in the form of epitaxial $^{1,8}$ polycrystalline $^{5-7}$ patterned films. These structures have shown novel properties and are a new tool to test micromagnetic predictions; however, in general, a hardening of the magnetic material is observed as the lateral dimensions decrease below $1 \mu \mathrm{m}$, so that the coercive and saturation fields of the structures increase by at least one order of magnitude respect to the unpatterned material, presenting typical values easily above a hundred Oersted. ${ }^{8,9}$ This result could be an undesirable effect to fabricate certain devices for applications. The material crystalline microstructure with its particular defects is an important factor that could play a role in this behavior; for example, when the pattern dimensions are reduced to the $100 \mathrm{~nm}$ range, the grain size can become relevant influencing the magnetization reversal processes. For these reasons, it is interesting to prepare submicrometric structures of amorphous magnetic materials. Their high density of defects and disorder results in an effective averaging in a scale much smaller than the minimum accessible pattern size.

In this work, we have fabricated submicrometric structures of amorphous $\mathrm{Fe}_{x} \mathrm{Si}_{1-x}$ in order to obtain magnetic arrays with low coercivity. The compositional dependence of the coercive field in the unpatterned samples has been analyzed in our system to carefully select the lowest values, so that the magnetic behavior of the submicrometric patterns is optimized to be as soft as possible.

\section{RESULTS AND DISCUSSION}

$\mathrm{Fe}_{x} \mathrm{Si}_{1-x}$ thin films have been obtained by dc magnetron sputtering on $\mathrm{Si}(100)$ substrates. The samples are grown at room temperature with a sputtering $\mathrm{Ar}$ pressure of $10^{-3}$ mbar (the system base pressure is $10^{-8}$ mbar) up to a film thickness of $40 \mathrm{~nm}$. The alloy is prepared from high purity $\mathrm{Fe}$ and $\mathrm{Si}$ targets, and the composition is varied by controlling the relative power of the sputtering guns. The final composition has been calibrated using $\mathrm{x}$-ray microanalysis. Also, the sample microstructure has been analyzed by x-ray diffraction to check the absence of crystalline phases in the films; only for Fe contents above $x=0.8$ diffraction peaks corresponding to polycrystalline $\mathrm{Fe}$ have been detected in the samples, in good agreement with previous results. ${ }^{10}$

The magnetic behavior has been characterized by magneto-optical transverse Kerr effect (TKE) measurements in a experimental setup reported elsewhere. ${ }^{11}$ The hysteresis loops reveal the presence of an uniaxial magnetic anisotropy. The compositional dependence of the coercive field $\left(H_{C}\right)$ measured along the easy axis is shown in Fig. 1. With increasing Fe content, $H_{C}$ decreases by an order of magnitude down to a minimum value $H_{C}=1.2 \mathrm{Oe}$ in the range $x$ $=0.7-0.75$. For $x=0.8$ the coercive field increases again due to the presence of polycrystalline phases. Figure 1(b) shows the anisotropy fields $H_{K}$ of the samples as a function of $\mathrm{Fe}$ content, measured by transverse susceptibility. It is 

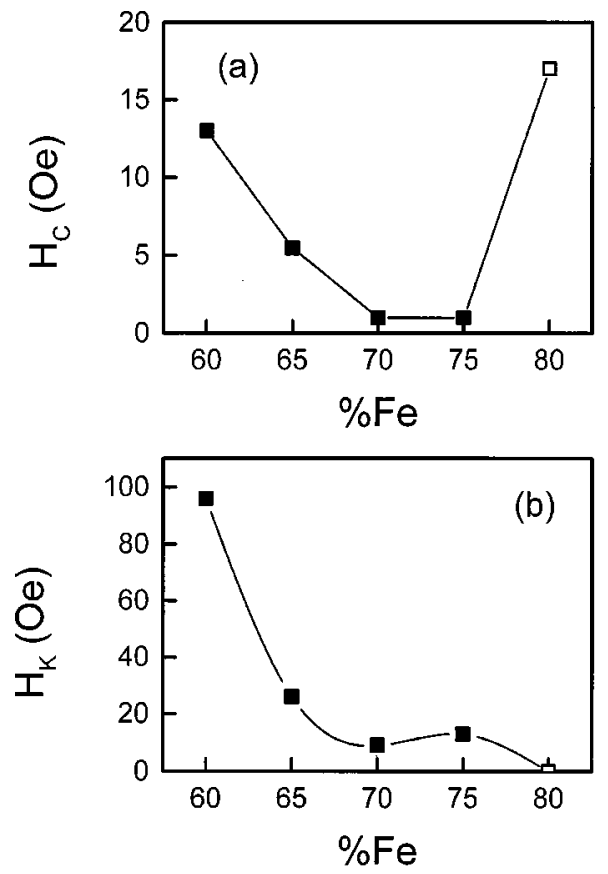

FIG. 1. (a) Compositional dependence of the coercive field along the easy axis of $40 \mathrm{~nm}$ thick $\mathrm{Fe}_{x} \mathrm{Si}_{1-x}$ films. (b) Compositional dependence of the anisotropy field measured by transverse susceptibility of $40 \mathrm{~nm}$ thick $\mathrm{Fe}_{x} \mathrm{Si}_{1-x}$ films. Filled symbols indicate amorphous samples and hollow symbols correspond to a film with polycrystalline phases.

found that, in the amorphous samples, the uniaxial anisotropy is the weakest for $x=0.7\left(H_{K}=9 \mathrm{Oe}\right)$. Therefore, in order to prepare the submicrometric structures a composition $\mathrm{Fe}_{0.7} \mathrm{Si}_{0.3}$ has been selected.

Electron beam lithography combined with a liftoff technique has been used to prepare the patterned magnetic arrays. ${ }^{12,13}$ Briefly, the Si substrates are first covered with a $300 \mathrm{~nm}$ thick electron sensitive PMMA resist layer; then, the pattern is defined with the electron beam of a Hitachi S-800 scanning electron microscope (SEM), so that, holes are made in the exposed regions of the PMMA resist after the sample is developed. Finally, a $40 \mathrm{~nm}$ thick $\mathrm{Fe}_{0.7} \mathrm{Si}_{0.3}$ film is grown on top of this resist template and the submicrometric structure is obtained by a liftoff process in acetone. In this way, arrays of lines have been obtained over areas of $250 \mu \mathrm{m} \times 250 \mu \mathrm{m}$ with typical spacings of $1 \mu \mathrm{m}$ and linewidths below $500 \mathrm{~nm}$. Figure 2 shows a SEM image of one of these arrays of $\mathrm{Fe}_{0.7} \mathrm{Si}_{0.3}$ lines. In this case, the well straight and parallel lines are $180 \mathrm{~nm}$ wide and are separated by $1 \mu \mathrm{m}$.

The TKE hysteresis loop of an array of $\mathrm{Fe}_{0.7} \mathrm{Si}_{0.3}$ lines with $H$ field parallel to the lines is shown in Fig. 3, where it is compared with the loop of a reference unpatterned film of the same composition. Previous results in similar structures of pure transition metals or $\mathrm{NiFe}$ alloys show hysteresis loops with typical coercive and saturation fields above 100 Oe; for example, Shearwood et al. ${ }^{8}$ find $H_{C} \approx 500$ Oe in 1 $\mu \mathrm{m}$ spaced lines made of epitaxial $\mathrm{Fe}(001)$, and Adeyeye et al. ${ }^{9}$ obtain values of $H_{C} \approx 200 \mathrm{Oe}$ in $2 \mu \mathrm{m}$ separated policrystalline $\mathrm{Ni}_{0.8} \mathrm{Fe}_{0.2}$ lines. In our case, the magnetic behavior in the array of $\mathrm{Fe}_{0.7} \mathrm{Si}_{0.3}$ lines is also harder than in the un-

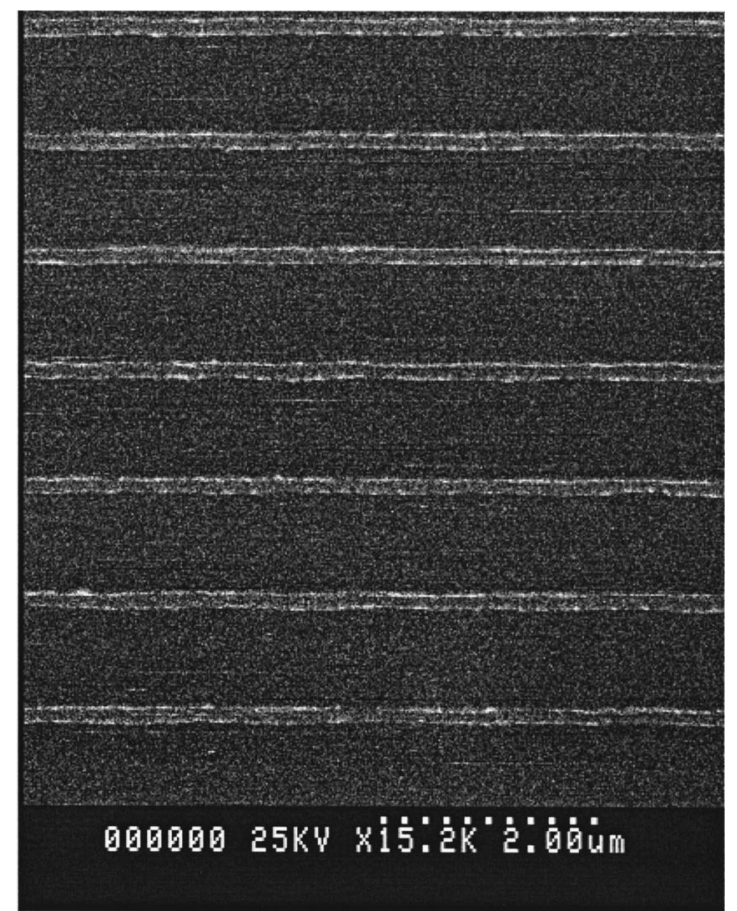

FIG. 2. SEM image of an array of $\mathrm{Fe}_{0.7} \mathrm{Si}_{0.3}$ lines prepared by electron beam lithography. The lines are $180 \mathrm{~nm}$ wide, $250 \mu \mathrm{m}$ long, and spaced by $1 \mu \mathrm{m}$.

patterned reference sample, with an increase of $H_{C}$ of almost one order of magnitude. But the observed coercive field $H_{C}=9$ Oe is still much smaller than the previously reported values in the literature with other materials. The saturation field $\left(H_{S}\right)$ of 40 Oe is also relatively small; however, as the magnetic field is tilted away from the direction parallel to the lines, the saturation field increases sharply. This must be related to the high value of the demagnetizing field $\left(H_{D}\right)$ in
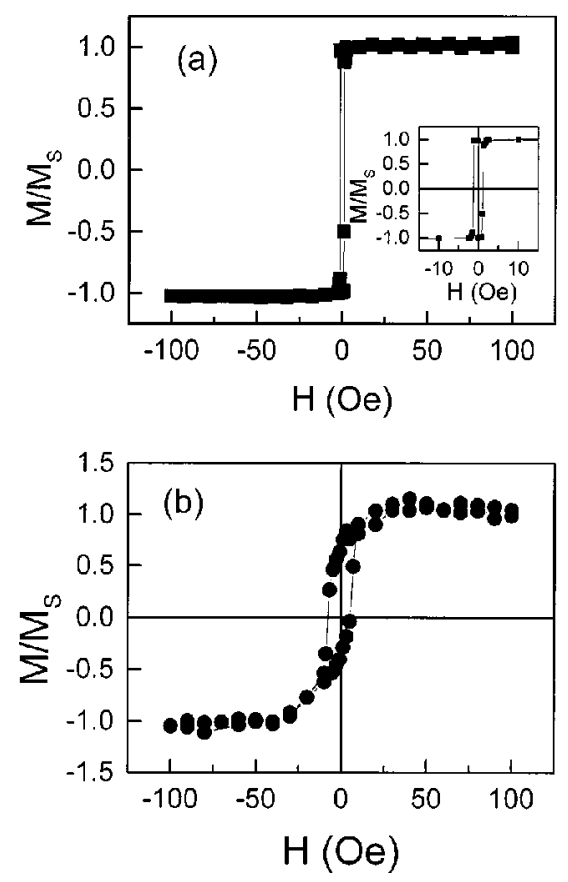

FIG. 3. TKE hysteresis loops of (a) unpatterned $\mathrm{Fe}_{0.7} \mathrm{Si}_{0.3}$ reference film, (b) array of $\mathrm{Fe}_{0.7} \mathrm{Si}_{0.3}$ lines $180 \mathrm{~nm}$ wide, $250 \mu \mathrm{m}$ long, and spaced by $1 \mu \mathrm{m}$. 
the direction perpendicular to the lines, which can be estimated $^{14}$ as $H_{D}=M_{S} * t \alpha(s / w) / w=2510 \mathrm{Oe}$, where $M_{S}$ is the saturation magnetization, $t$ is the film thickness, $w$ is the linewidth, $s$ is the interline distance and the function $\alpha(s / w) \approx 1$ in our line geometry. It is interesting to note that as the width of the lines is smaller than the line spacing, this kind of structure can be considered as an array of noninteracting wires. ${ }^{14}$ Actually, preliminary results in our samples indicate that there is not any significant change in the coercive field as the interline distance is reduced from $1 \mu \mathrm{m}$ to $0.6 \mu \mathrm{m}$.

\section{CONCLUSIONS}

In summary, submicrometric structures of amorphous FeSi have been prepared by electron beam lithography combined with a liftoff technique. After carefully optimizing the composition to obtain the softest magnetic behavior, coercive field values as low as 9 Oe can be observed in arrays of noninteracting $\mathrm{Fe}_{0.7} \mathrm{Si}_{0.3}$ lines with $180 \mathrm{~nm}$ width. These values are one order of magnitude smaller than previously reported coercive fields for similar structures made of polycrystalline and epitaxial magnetic materials.

\section{ACKNOWLEDGMENTS}

Work supported by Spanish CICYT (Grant No. PB941351 and Grant No. MAT96/904).

${ }^{1}$ M. Hehn, K. Ounadjela, J. P. Bucher, F. Rousseaux, D. Decanini, B. Bartenlian, and C. Chappert, Science 272, 1782 (1996).

${ }^{2}$ J. P. Spallas, A. M. Hawryluk, and D. R. Kania, J. Vac. Sci. Technol. B 13, 1973 (1995).

${ }^{3}$ D. D. Awschalom, M. A. McCord, and G. Grinstein, Phys. Rev. Lett. 65, 783 (1990).

${ }^{4}$ J. F. Smyth, S. Schultz, D. R. Fredkin, D. P. Kern, S. A. Rishton, H. Schmid, M. Cali, and T. R. Koehler, J. Appl. Phys. 69, 5262 (1991).

${ }^{5}$ R. D. Gomez, M. C. Shih, R. M. H. New, R. F. W. Pease, and R. L. White, J. Appl. Phys. 80, 342 (1996)

${ }^{6}$ M. S. Wei and S. Y. Chou, J. Appl. Phys. 76, 6679 (1994).

${ }^{7}$ T. Taniyama, I. Nakatani, T. Namikawa, and Y. Yamazaki, Phys. Rev. Lett. 82, 2780 (1999).

${ }^{8}$ C. Shearwood, S. J. Blundell, M. J. Baird, J. A. C. Bland, M. Gester, H. Ahmed, and H. P. Hughes, J. Appl. Phys. 75, 5249 (1994).

${ }^{9}$ A. O. Adeyeye, J. A. C. Bland, and C. Daboo, J. Magn. Magn. Mater. 188, L1 (1998).

${ }^{10}$ J. A. Aboaf, R. J. Kobliska, and E. Klokholm, IEEE Trans. Magn. MAG14, 941 (1978).

${ }^{11}$ L. M. Alvarez-Prado, G. T. Perez, R. Morales, F. H. Salas, and J. M. Alameda, Phys. Rev. B 56, 3306 (1997).

${ }^{12}$ J. I. Martín, Y. Jaccard, A. Hoffmann, J. Nogues, J. M. George, J. L. Vicent, and I. K. Schuller, J. Appl. Phys. 84, 411 (1998).

${ }^{13}$ J. I. Martín, J. L. Vicent, J. V. Anguita, and F. Briones, J. Magn. Magn. Mater. 203, 156 (1999).

${ }^{14}$ A. O. Adeyeye, J. A. C. Bland, C. Daboo, and D. G. Hasko, Phys. Rev. B 56, 3265 (1997) 